\title{
SM EVM Control Computer Development
}

\author{
N.L. Prokhorov ${ }^{1}$ and G.A. Egorov ${ }^{2}$ \\ ${ }^{1}$ Supervisor of Studies, INEUM, Moscow, Russia \\ prokhorov_n@ineum.ru \\ ${ }^{2}$ Deputy General Director, INEUM, Moscow, Russia \\ egorov_g@ineum.ru
}

\begin{abstract}
The main stages in the development of the Institute of Electronic Control Computers during fifty years of its existence are considered in this paper. The basic models of controlling computer systems, developed at the institute are presented. Creating and large-scale industrial production of smallcomputer system SM EVM have founded the technological base of automation control and information processing in various branches of industry of the country.
\end{abstract}

Keywords: INEUM, control computers, SM EVM, real-time control.

\section{Introduction}

One of the major development paths of the national computer engineering is associated with the Institute of Electronic Control Computers (INEUM), organized by its first director, one of the pioneers of the national computer engineering, Corresponding Member of the USSR Academy of Sciences I.S. Bruk (1902 - 1974).

As the school of Academician S.A. Lebedev was oriented toward designing maximum-throughput computers for each generation of elements, I.S. Bruk's conception from the very beginning was vectored to the class of small and medium computers for which the cost/performance index and balanced characteristics are extremely important. It is interesting to state that both S.A. Lebedev and I.S. Bruk proceeded to design digital computers on the background of the experience obtained by using the analog computers in the area of power engineering and deep understanding of its disadvantages.

\section{Virtues of I.S. Bruk}

The first Soviet inventor certificate for the digital computer was received by I.S. Bruk and B.I. Rameev with priority of December 1948. The automatic digital computer M1 was designed at the Laboratory of Electrical Systems of the Energy Institute of the USSR Academy of Sciences in 1950-1951 under the guidance of I.S. Bruk. It was brought into service in 1952, several months later than the MESM computer designed by S.A. Lebedev in Kiev. The main concepts of M-1 were formulated by I.S. Bruk and N.Ya. Matyukhin, who was then a young graduate of the Moscow Power Institute 
(MEI) and future Corresponding Member of the USSR Academy of Sciences. The M1 realized the two-address instruction system and some important decisions concerning selection of logic and circuitry of the digital computers, which played an important role in the future development of the national computer engineering.

It is important to notice here that the designers of MESM and M-1 arrived to the classical design of their digital computers based on the stored-program (von Neumann) architecture independently of each other. The works of the American scientists were known in the USA since 1946, but first published in an abbreviated form in 1962.

In the laboratory of I.S. Bruk in 1952, a group of MEI graduates headed by M.A. Kartsev developed the M-2 computer. Working at approximately the same speed as the "Strela" computer, M-2 had four times less number of electronic tubes, consumed seven to eight times less power and occupied one-tenth the area. These achievements were due to the use of conventional oscillographic cathode-ray tubes as main-memory elements and semiconductor diodes in the logic circuits. It seems that it was for the first time that at upgrading M-2 in 1953-1956 M.A. Kartsev realized the idea of shortened instruction addresses (with switching of the memory areas) and shortened operation codes as means of coordinating the instruction and number formats. This idea preceded the methods of generating the execution addresses in the computers of the second and third generations.

Based on the experience gained in the work on M-1 and M-2, I.S. Bruk formulated the concepts of "small-sized" computers. The first solution of this problem was embodied in M-3 designed 1956 in collaboration with LUMS of the USSR Academy of Sciences (I.S. Bruk) and NIIEP (Academician A.G. Iosif'yan). The M-3 philosophy was formulated by I.S. Bruk, N.Ya. Matyukhin, V.V. Belynskii, B.M. Kagan, and V.M. Dolkart. M-3 served as a prototype of two commercial families of domestic computers realized by the designers of M-3: "Minsk" (G.P. Lopato, later Corresponding Member of the USSR Academy of Sciences) and "Razdan" (B.B. Melik-Shakhnazarov, Institute of Mathematics of the Armenian Academy of Sciences from which the Yerevan Institute of Mathematical Machines was later separated).

\section{The INEUM Era}

Establishment of INEUM was preceded in 1957 by formulation by I.S. Bruk of the scientific problem "Development of the Theory, Principles of Design and Application of the Special-Purpose Computing and Controlling Machines." The problem report compiled by an expert team headed by I.S. Bruk was published by the USSR Academy of Sciences in 1958 in the serial edition "Problems of Soviet Science". The report indicated to the significance of the control machines for the national economy and, for the first time, presented and substantiated the main directions of fundamental and applied studies in the field of production automation and plant control by specialpurpose and control machines.

In the course of time, the notion of special-purpose control and computing machines as formulated in the report with regard to the first-generation computers changed its initial significance owing to the rapid progress of electronics. At the same time, the I.S. Bruk's notion of the "control computers" which are distinguished from 
the general-purpose computers in the nature of their relation with the control plant, higher reliability, real-time operation, ability to work in unfavorable industrial environment, and so on retained its value and was further developed.

In 1957 a team of I.S. Bruk's disciples headed by N.Ya. Matyukhin joined a defense research institute to design digital computing facilities for the air defense systems. In 1957, while staying with INEUM, N.Ya. Matyukhin independently of M. Wilkes proposed the principle of the microprogram computer control that required detailed working out of the algorithms of arithmetic operations, their decomposition into elementary micro-operations and prototyping of the circuits of the microprogram computer with microprograms stored in the read-only memory. This principle was then widely used in computers both in INEUM and elsewhere by the designers of control and other computers.

In 1958-1964 INEUM developed the control computer M4 (M4-M, M4-2M) intended for real-time control of a radar complex (Radiotechnical Institute of the USSR Academy of Sciences, Academician A.L. Mints) incorporated in the radioelectronic system of Earth satellite observation. M4 was one of the first domestic computers based on the second-generation circuitry. Although the decision about commercial production of M4 (M4M) was made in 1962 after successful tests on the working prototype of the radar complex, M.A. Kartsev, the Chief Designer of M4 insisted on its substantial updating in view of the fact that the progress of electronics in 1958-1962 would enable dramatic enhancement of the computer performance, thus making it one order of magnitude more powerful than the thenmanufactured Soviet computers. The modernized M4-2M computer had speed of 220 thousand operations per second on programs stored in the read-only memory, main memory up to $16 \mathrm{~K}$ words (29 digits), memory of instructions and constants up to $12 \mathrm{~K}$ words, (29 digits). This variant of M4-2M was commercially produced during fifteen years since 1964. In 1968 peripheral computers M4-3M were designed for it with the aim of inputting and preprocessing the arriving data, storaging, documenting, and outputting information to the external users at simultaneous asynchronous operation of all user's systems and facilities. The complex of M4-2M and M4-3M had speed of about 400 thousand operations per second.

The head of these designs, M.A. Kartsev, made a substantial contribution to the development of the national digital computers and control computers. He generalized his experience in the monograph "Architecture of Digital Computers" published in 1978 as applied to the third-generation computers.

In 1967, M.A. Kartsev proposed a new approach to architecture and structure of the computer systems based on parallel computations. A draft design of the computer system M-9 with power of 1 billion operations per second was designed in INEUM under the leadership of M.A. Kartsev. Operations over a new class of operands-not numbers but functions of one or two variables defined at discrete points-had to be executed in M-9 on the $32 \times 32$ matrix of computing elements with common instruction flow. The project of M-9 was rich with new ideas, but many of them have not been implemented until now. Later, M.A. Kartsev's team formed the kernel of a research institute of computer systems bearing now his name. This institute developed high-performance computer systems M-10 and M-13 realizing some concepts suggested in the project of M-9. 
The general-purpose computer M-5 intended for planning and economical calculations was developed in 1958-1961 in INEUM under the leadership of I.S. Bruk. It was distinguished for the advanced possibilities of multiprogram and multiterminal operation and, being one of the first domestic computers based on the second-generation circuits and to a large extent was an architectural and structural predecessor of the third-generation computers. It deserves noting that during the development work the INEUM designers of M-5 had no information about the existing computers having the same potentialities. Information about the foreign multiprogram computers such as "Atlas," "Gamma-60," and others became known in this country much later when the design of M-5 was completed.

Automation of powerful energetic "boiler-turbine-generator" blocks at the thermal stations was an important line of INEUM's work in the 1960's. The control computers M-7 designed at INEUM were put in operation at a $200 \mathrm{MW}$ block of the Shchekino station in 1966 and in 1969 at the 800 MW block of the Slavyanskaya station. The M-7-based block control systems maintained normal operation of the blocks and optimized their operation by minimizing the fuel consumption and determining the appropriate setups of the controllers, as well as executed complex logical programs of block startup and shutdown, analysis of combinations of the block parameters with the aim of detecting the pre-emergency situations, and display to the operator of the required information. Design and introduction of M-7 were headed by N.N. Lenov and N.V. Pautin who was the INEUM director in 1964-1967.

In 1965 INEUM headed the project of the USSR Ministry of Instrumentation on the development of the Aggregate system of Microelectronic Facilities of Computer Engineering (ASVT-M) intended primarily for process automation in industry and automatic control systems of enterprises. Although at that time production of the domestic integral circuits was at the stage of development and experimental use, the first control computer systems (UVK) of the third generation were developed in INEUM already in 1970. These systems provided the groundwork of the automated systems that were repeatedly designed in the 1970s for automation of supervision in large power distribution systems, process and enterprise control in machine building, metallurgy, and other industries, as well for automation of research and experiments. B.N. Naumov (1927-1988) who headed INEUM in 1967 (elected in 1984 the Member of the USSR Academy of Sciences) was appointed the Chief Designer of ASVT-M.

The philosophy, structure, and principles of unification of the UVK models, assemblies and devices of ASVT were developed in the late 1960' jointly by INEUM (E.N. Filinov) and NIIUVM (V.V. Rezanov).

The two following basic considerations were taken into account at determination of the principles of architecture and structure of the models of computers and UVK included in ASVT:

o the need for a whole number of models for several hierarchical levels of the industrial automatic control systems satisfying the requirements of problems of different classes such as centralized monitoring of the process parameters, local control of individual technological plants and machinery, supervisory control of manufacturing, planning and so on;

$\circ$ the possibility of designing general-purpose control computers and systems based on circuitry of the second and third generations instead of the numerous 
dedicated computers that were designed in the 1950s and early 1960s. The premises for formation of the ASVT philosophy were based on the preceding experience gained by NIIUVM at designing the computer systems of SOU-1 family, Institute of Cybernetics of the Ukrainian Academy of Sciences ("Dnepr" computer), INEUM (M-4, M-5, M-7 computers).

\section{New Directions}

An architecture supporting program compatibility with the models of the uniform system of computers that were produced commercially in the USSR from the early 1970's was selected for the upper-level models (M-2000, M-3000, M-4000/M-4030). Elaboration of the interface of the upper-level ASUP models to the control systems of lower levels that was carried out within the framework of ASVT paved the way for various projects of joint use of the uniform system of computers and SM EVM. At developing the ASVT software, the problem of compatibility of allied architectures at the level of the operating systems was solved. Being application-compatible with the Siemens OS BS-2000 (architecture of the Siemens 4004 family), the DOS ASVT operating system developed in INEUM under the leadership of I.Ya. Landau and V.A. Kozmidiadi ran on the M-4030 hardware that was binary compatible with the IBM 360/370 and the uniform system of computers (ES EVM). The M-4000/M-4030 hardware was developed at INEUM under the guidance of V.G. Zakharov. M-2000 was produced commercially by the Severodonetsk Instrumentation Plant, and M-3000 and M-4030 by the Kiev Plant of control computers.

Two architectural directions were selected for the medium-level ASVT-M models belonging to the class of mini-computers. The first direction was represented by the models M-6000 and M-7000 developed at NIIUVM under the guidance of V.V. Rezanov and V.M. Kostelyanskii and produced by the Severodonetsk Instrumentation Plant and Kiev and Tbilisi Plant of control computers.

The second architectural direction of the medium-level ASVT-M models was represented by $\mathrm{M}-400$, which also belonged to the class of mini-computers. Its architecture had instruction set and addressing mode that were software-compatible with the PDP-11 family of mini-computers of Digital Equipment Corp., which at that time was internationally recognized as a de facto standard, as well as the Unibus system interface to which the peripheral controllers (external memory, I/O) were connected and the controller interfacing the host computer to the object communication devices (USO) from the USO M-6000/M-7000 nomenclature and with the M-40 centralized monitoring machines.

\section{Use of CAD}

The possibility of connecting the controllers of the hardware connected to the instrumentation interfaces to the Unibus enabled one to design M-400-based problemoriented measurement-and-computation systems (IVK) using facilities satisfying the CAMAC (Computer-aided Measurement and Control) international standards and ASET (Aggregate Complex of Electrical Measuring Instrumentation). This made 
ASVT-M applicable not only in the field of industrial automation, but also in automation of research and experimentation.

Computer-aided design (CAD) became a pivotal application of M-400 which underlay the problem-oriented systems in the form of automated workplaces (ARM) for the radio-electronic and machine building CAD systems including the necessary graphic devices of information I/O and the dedicated software. The Interdepartmental Program for CAD Systems in the Defense Industries headed by the Ministry of the Radio Industry of the USSR and its head organization, the "Almaz" research institute also exemplified formation and implementation of the state policy in science and technology. In these works, the INEUM was represented by I.Ya. Landau and E.N. Filinov. M-400 was commercially produced since 1974 by the Kiev Computer Plant, IVK was produced by the Vilnius Plant of Electrical Instrumentation, and ARM, by the Gomel Plant of Radiotechnical Equipment (GZRTO).

Development in the first half of the 1970s within the framework of ASVT-M of the efficient interactive graphical displays oriented to the architecture of the Unibus computers enabled use of the INEUM achievements in the CAD systems. The characteristics of the M-400-based graphic system and display graphic console EPG400 (V.I. Fuks) allowed the Central Design Bureau "Almaz" (Academician B.V. Bunkin) to use it as the main platform of the automated designer workplaces for the defense-complex enterprises. Integration of ARM's based on M-400 and EPG-400 was done at GZRTO, which enabled fast introduction of ARM's in the practice of design.

A special place within ASVT-M was occupied by the model M-5000, which also was classified as mini-computers. It was intended to replace the obsolete punch card machines at the mechanized accounting offices of the USSR Central Statistical Department. The original architecture of M-5000 allowed its specialized application to accounting and statistical tasks. M-5000 was designed and commercially produced by the Vilnius Plant of Accounting Machines (Chief Designer A.M. Nemeikshis).

The centralized monitoring and control computer M-40 occupying the lower level in the hierarchy of the ASVT-M models was intended for acquisition, preprocessing, and logging of the process parameters, multichannel two-position control and information output to the digital indicators and CRT's of the operator consoles. M-40 had 1688 (1000 analog and 688 digital) input channels, sensor interrogation speed of 400 channels per second, error of measurement of analog signals of $0.4 \%$. The number of two-positional control channels was 960. M-40 used the microprogram principle of execution of the programs stored for higher reliability in a 16 Kbyte readonly memory. M-40 was designed at INEUM (E.V. Keshek, N.D. Kabanov) and produced commercially by the Moscow "Energopribor" plant (S.Ya. Lebedinskii, V.P. Fedorin).

\section{Birth of SM EVM}

By a decision of the Intergovernmental Commission on Cooperation of the Socialist Countries on Computers (MPK for VT), in 1974 INEUM was nominated the head organization for design to the small-computer system (SM EVM) and its director B.N. Naumov was appointed the Chief Designer of SM EVM. Since 1984, 
N.L. Prokhorov became the director of INEUM and Chief Designer of SM EVM. The complex of SM EVM R\&D was carried out by more than thirty institutes and enterprises of the USSR, Bulgaria, Hungary, GDR, Cuba, Poland, Rumania, and Czechoslovakia.

SM EVM included a set of the basic models of micro- and minicomputers such as the basic series of processors of various performance and main memories, wide nomenclature of the I/O facilities, external memory, information display, interface to the object, intra-machine and inter-machine communication. SM EVM was intended for control computer complexes used in the control systems of industrial processes and installations, measurement and computation complexes included in the CAD systems, complexes of data acquisition and processing in the industrial control systems, as well as for small-size commercial and engineering calculations. Since the mid-1970s two mutually complementing international systems of uniform computers and SM EVM became the technical groundwork of automation of control and information processing in all national industries of the participants of the agreement on cooperation in computer engineering.

The advanced technological principles and standards of SM EVM encompassed all aspects of unification of elements, assemblies and devices, designs, models, computers, software with allowance for the technology and power of the national industry and enabled organization of large-scale production on the basis of cooperation of specialized enterprises of various countries.

The design of SM EVM relied on general principles, some of which deserve special mentioning:

o provision of continuity with the previous ASVT-M computers and models:M-400 (SM 3, SM 4, SM 1300, SM 1420), M 5000 (SM 1600),M 6000/7000 (SM -1, SM -2, SM 1210, SM 1634), "MIR" (SM 1410);

o design of systems with task sharing on the basis of general-purpose and dedicated processors of SM EVM;

o wide use of the microprogram control for implementation of the main functions of processors and controllers;

o use of the programmable controllers of the peripherals;

o common nomenclature of the peripherals for some models thanks to the standard interfaces of the peripherals;

o advanced nomenclature of the data-transmission adapters for interfacing the SM EVM computers with the communication lines in compliance with the international standards;

o interfaces of SM EVM to the uniform system computers in heterogeneous systems and networks;

o design of problem-oriented systems produced on the basis of the SM EVM models: measurement and computation systems (IVK) with the CAMAC or ASET GSP hardware, automated workplaces (ARM) for the CAD systems in machine building, radioelectronics, construction engineering, and so on;

o unique form-factors for all SM EVM facilities in compliance with the standards of the International Electrotechnical Commission. 
The SM EVM-based IVK of CAMAC or ASET were oriented to real-time automation of complex experiments in various fields of science and technology. Flexibility and modularity of the SM EVM facilities, advanced facilities for interfacing the computer and experiment, problem-oriented system and applied SM EVM software enabled wide use of IVK in the systems of research automation, primarily at the institutions of the USSR Academy of Sciences.

The advent of SM EVM enabled a fundamental enhancement of efficiency and generality of the use of automated workplaces in CAD systems. The potentialities of the universal basic graphical and applied software of the database control systems made real the interactive design, convenient representation of the results, input, editing and output of graphics, diagrams, and drawing. The CAD systems included graphic periphery developed by the defense enterprises for radioelectronic applications (ARM-R), machine building (ARM-M), construction (ARM-S), economics (ARM-E), and so on. Realization of the principle of firmware compatibility of all SM EVM facilities enabled easy successive improvement of the ARM performance by adding the SM 3, SM 4, SM 1420, SM 1700 processors and the graphical vector and raster displays EPG-SM and EPG-3 developed at the institute. (V.I. Fuks).

\section{SM EVM Architectures}

The design of SM EVM followed two architectural lines. The first line included a wide nomenclature of the control computer systems based on the micro-computers of the SM 1800 family constructed using the bus-model principle (N.L. Prokhorov, A.N. Shkamarda, N.D. Kabanov, A.Ya. Sokolov).

The first models of this line represented 8-bit microcomputers (microprocessor KR580) relying on the bus-model principle with the internal interface I41 (Multibus). In 1986 the first 16-bit model SM 1810 (microprocessor K 1810) of this family was designed, and its manufacture started. All in all, six general-purpose modifications of the SM 1810 and four modifications adjusted to the industrial environment (SM 1814) were developed.

Development of the 32-bit computer system SM 1820 based on the Intel 80386 microprocessor was completed in 1990. All in all, 26 modifications of the SM 1800 family were designed and produced commercially.

A wide nomenclature of external devices, intercouplers to the object, network facilities, adapters and various interfaces (C2, RS422, ILPS, BITBUS, IRPR, and so on) was developed within the framework of this line.

All developments of the SM 1800 family accepted and realized the bus-modular architecture, which enabled virtually continuous evolution of all models of the family both in terms of enhanced performance and satisfaction of the functional requirements of diverse applications.

The system software of SM 1800 included instrumental, real-time executive, and general-purpose operating systems.

Production Associations "Elektronmash" (Kiev) and K.N. Rudnev Plant of control computers (Orel) were the subcontractors at all stages of the development of the SM 1800 family. 
The second architectural line of SM EVM was represented by a number of software-compatible models of mini-computer of various performances. The minor models of this line included the 16-bit Unibus-based computers SM 3, SM 4, SM 1300, and SM 1420 (INEUM - B.N. Naumov, A.N. Kabalevskii, V.P. Semik, Yu.N. Glukhov, E.N. Filinov; "Elektronmash" - V.A. Afanas'ev, S.S. Zabara, V.G. Mel'nichenko, V.N. Kharitonov).

SM 1420 then was elaborated to the SM 1425 computer system (N.L. Prokhorov, L.M. Plakhov, G.A. Egorov) using a 22-bit parallel bus system interface featuring more advanced architectural potentialities.

A special place in this architectural line was occupied by the 32-bit mini-computers of the SM 1700 with the Unibus interface and SM 1702 with MPI interface (Chief Designer N.L. Prokhorov, V.V. Rodionov, V.I. Frolov, G.A. Egorov, L.M. Plakhov). The architecture of this family supported virtual memory, software and hardware compatibility with the 16-bit models of mini-computers, as well as an advanced diagnostic system.

The software of this line was represented by a wide spectrum of operating systems, network software for local-area and distributed networks, packages of diverse applied programs.

At developing the SM EVM architecture, original principles of systems with function sharing were elaborated. Owing to that the designers using the circuitry succeeded in implementing two-processor computer systems that were softwarecompatible with the earlier "MIR" computers for engineering calculations and computers of the M 5000 line for commercial applications.

Controllers and peripheral devices, as well as specially designed processors enhancing substantially the throughput on a particular class of problems occupy an important place in the nomenclature of SM EVM. First of all, the specially designed processor for fast Fourier transforms developed in collaboration with the Institute of Radiotechnics and Electronics of the USSR Academy of Sciences for processing the radar images of the Venus surface (B.Ya. Fel'dman) deserves mentioning. This largescale study carried out by the USSR Academy of Sciences under the guidance of Academician V.A. Kotel'nikov required computer power equivalent to supercomputers. However, the problem was resolved using a mini-computer equipped with a specially designed Fourier processor.

The logic modeling processor representing a dedicated computer for rapid modeling of digital circuits (B.G. Sergeev) deserves special mentioning. Its domain of application is the CAD systems for VLSI circuit design. Original data-flow (pipeline) architecture of the specially designed processor enabled approximately a thousandfold acceleration of modeling as compared with the general-purpose computers.

\section{Conclusion}

The aforementioned data about the SM EVM family show that it did not copy the foreign prototypes, relied on the domestic circuits, and was software-compatible with the family of mini-computers that were then most popular in the West. This aim was well justified because otherwise the national computer engineering would be isolated from the international progress in this area and, in particular, would be unable to get access to the accumulated internationally software. 
Over sixty thousand computers and control systems of SM EVM, as well as measurement and computer systems (IVK), and automated workplaces (ARM) based on SM EVM were produced from 1974 to 1990 using the INEUM developments.

Importantly, the SM EVM industry had a nationwide infrastructure for technical servicing and training. The SM EVM facilities were a popular school for tens of thousands of experts that were then entering the world of the computer technology. 